\title{
Récits non naturels, narratologie non naturelle : apports, problèmes et perspectives
}

Unnatural Narratives, Unnatural Narratology: Contributions, Problems and

Perspectives

\section{Sylvie Patron}

\section{OpenEdition}

\section{Journals}

Édition électronique

URL : http://journals.openedition.org/pratiques/5726

DOI : 10.4000/pratiques.5726

ISSN : 2425-2042

Éditeur

Centre de recherche sur les médiations (CREM)

Référence électronique

Sylvie Patron, «Récits non naturels, narratologie non naturelle : apports, problèmes et perspectives », Pratiques [En ligne], 181-182 | 2019, mis en ligne le 30 juin 2019, consulté le 22 juillet 2019. URL : http://journals.openedition.org/pratiques/5726; DOI : 10.4000/pratiques.5726

Ce document a été généré automatiquement le 22 juillet 2019.

(c) Tous droits réservés 


\section{Récits non naturels, narratologie non naturelle : apports, problèmes et perspectives}

Unnatural Narratives, Unnatural Narratology: Contributions, Problems and Perspectives

Sylvie Patron

L'étude des récits non naturels (unnatural narratives), ou narratologie non naturelle (unnnatural narratology), est l'un des derniers courants apparus au sein de la narratologie postclassique ${ }^{1}$. Cette approche parait novatrice et prometteuse car elle rejoint plusieurs préoccupations actuelles associées à la théorie, à l'analyse et à l'interprétation des récits de fiction. Elle est aussi intensément discutée dans les milieux narratologiques. Loin d'être une approche unifiée, la narratologie non naturelle se compose de différentes tendances, basées sur des définitions légèrement différentes des récits non naturels, prônant des approches parfois très différentes, voire opposées, de ces récits. La narratologie non naturelle peut déjà se prévaloir de résultats importants dans l'étude des récits non naturels ou des éléments non naturels dans les récits, généralement négligés dans la ou les narratologies établies. Une étude un peu approfondie fait cependant apparaître certains problèmes. J'en donnerai un exemple significatif à la fin de cet article.

\section{La narratologie non naturelle : bref historique du mouvement}

2 Si l'on cherche des traces des discussions et éventuellement des démarches qui ont conduit à la naissance de la narratologie non naturelle, ce que l'on trouve d'abord concerne son nom. Un article de H. S. Nielsen publié en 2011 contient ces quelques lignes :

À l'issue du congrès annuel de l'[International Society for the Study of Narrative (ISSN)] à Washington en 2007, un petit groupe s'est trouvé réuni par hasard dans le 
lobby [de l'hôtel où avait lieu le congrès]. Il se composait de Brian Richardson, Jan Alber, Maria Mäkëlä et moi-même. Nous nous sommes rendu compte que ce qu'avait apporté le congrès était une résistance assez forte à l'égard des paradigmes naturalisants par ailleurs dominants. Nous avons donc invité toutes les personnes intéressées à faire partie d'un groupe consacré à ce que nous avons provisoirement appelé la « narratologie non naturelle ». (2011a, p. 55, n. 2, repris dans 2011b, p. 71) ${ }^{2}$

La «narratologie non naturelle» s'oppose à la narratologie «naturelle » (entre guillemets) de M. Fludernik (voir 1996)³. On a reproché par la suite aux narratologues non naturels de faire l'économie des guillemets. On leur a également fait remarquer que «non naturel(le) » induisait des connotations négatives ou stigmatisantes. Leurs réponses ont toujours été claires : la narratologie non naturelle est étrangère au débat entre nature et culture et ne désigne aucune pratique sociale ou aucun comportement comme naturels ou non naturels. Le terme «non naturel(le)» doit être compris dans le contexte de la recherche narratologique et n'a dans ce contexte que des connotations positives (voir Alber \& Heinze, 2011a, p. 2 ; Alber, Nielsen \& Richardson, p. 374 ; Richardson, 2012b, p. 98 ; Alber, Iversen, Nielsen \& Richardson, 2013b, p. 4 ; Richardson, 2015, p. 6 et 2016a, p. 393). À la suite de l'épisode du lobby, les narratologues non naturels ont acheté une adresse web, www.unnaturalnarratology.com ${ }^{4}$, qui a contribué à pérenniser le nom de la narratologie non naturelle.

La narratologie non naturelle a rapidement acquis une notoriété dans le monde académique: organisation de panels dans les congrès de l'ISSN et d'autres congrès internationaux ${ }^{5}$; publication d'un article-manifeste, « Unnatural Narratives, Unnatural Narratology: Beyond Mimetic Models ", signé de J. Alber, S. Iversen, H. S. Nielsen et B. Richardson (voir Alber et al., 2010) ${ }^{6}$. Actuellement, elle peut se prévaloir de deux ouvrages collectifs (Alber \& Heinze, 2011b ; Alber, Nielsen \& Richardson, 2013) ${ }^{7}$, de deux monographies (Richardson, 2015 ; Alber, 2016), auxquelles on peut ajouter l'ensemble des chapitres signés de B. Richardson dans Herman et al. (2012), de deux numéros ou parties de numéros de revue (Style, 2016 ; Storyworld, voir Richardson, 2017b) ${ }^{8}$, d'un dictionnaire en ligne (http://projects.au.dk/narrativeresearchlab/unnatural/undictionary), et on ne compte plus les articles individuels ou collectifs consacrés aux récits non naturels ou aux éléments non naturels dans les récits.

\section{Qu'est-ce qu'un récit non naturel ? Des définitions légèrement différentes}

B. Richardson (2015, p. 3, repris dans 2017a, p. 193 et 2018, p. 167) définit le récit non naturel comme "un récit qui contient des événements, personnages, cadres [spatiotemporels] ou schèmes [frames] significatifs antimimétiques. Par antimimétiques, [il] enten [d] des représentations qui vont à l'encontre des présuppositions des récits non fictionnels, violent les attentes mimétiques et les pratiques du réalisme, défient les conventions des genres existants, établis ${ }^{9}$. Reprenons ces différents termes. Mimétiques : qui se calquent sur ou ressemblent substantiellement à des récits non fictionnels. Les récits mimétiques cherchent à représenter le monde réel de référence d'une manière reconnaissable. Selon B. Richardson, la narratologie s'est construite à partir de ces récits et est affectée par un biais mimétique qui limite son pouvoir explicatif. Antimimétiques : qui rompent de différentes façons (ludiques, critiques, etc.) avec le projet ou le régime mimétique. B. Richardson introduit une distinction supplémentaire entre l'antimimétique 
et ce qu'il appelle le non-mimétique. Un récit antimimétique comme Molloy de S. Beckett défie les conventions de la représentation mimétique. Des récits non mimétiques comme les contes de fées, les fables animalières, les histoires de fantômes, etc., construisent un monde fictionnel cohérent, parallèle au monde réel de référence, avec ses lois propres, ses conventions, ses codes, ou dans certains cas ajoutent simplement des éléments surnaturels à une représentation qui reste par ailleurs mimétique du monde réel de référence. Seuls les récits antimimétiques créent un effet de surprise ou, selon le terme des formalistes russes, souvent cité par B. Richardson, de "défamiliarisation » (voir p. ex. Richardson, 2015, p. 24 et 2016b, p. 509). B. Richardson fait également remarquer que, si beaucoup de récits sont entièrement mimétiques, aucun ne peut être entièrement antimimétique. Les deux aspects sont présents simultanément, à différents degrés, dans de nombreux récits. Les récits mimétiques s'emploient généralement à masquer leur caractère artificiel (ou artefactuel); les récits antimimétiques, au contraire, attirent fréquemment l'attention sur leur statut de fiction, voire sur les procédés de leur composition. Les éléments antimimétiques (évènements, personnages, etc.) sont souvent plus remarquables et plus intéressants quand ils sont pris dans un rapport dialectique avec les aspects mimétiques du récit dans lequel ils apparaissent (voir Richardson, 2015, p. 4).

6 Dans la définition de B. Richardson, le non-naturel s'assimile donc à l'antimimétique. B. Richardson insiste notamment sur le fait que «[1]es œuvres non mimétiques conventionnelles ne sont pas, dans [sa] perspective, des récits non naturels » $(2015$, p. 4); ou selon une autre formulation, « [1]a fiction non naturelle n'est pas seulement différente de la fiction mimétique mais également de ce [qu'il] appelle la fiction non mimétique [... ]» (2016a, p. 386). Les exemples paradigmatiques de récits non naturels pour B. Richardson sont les récits non réalistes de J. L. Borges (p. ex. «L'Aleph »), L'Innommable de S. Beckett et La Jalousie d'A. Robbe-Grillet, qu'il mentionne souvent, Neige d'A. Kavan, Les Enfants de minuit de S. Rushdie, qu'il analyse en détail (Richardson, 2012a), ou encore Les Versets sataniques du même auteur. B. Richardson souligne que les récits non naturels se rencontrent dans presque toutes les périodes de l'histoire littéraire occidentale et qu'on peut également en trouver des équivalents dans d'autres cultures (voir 2015, chap. 5 et p. 5, 10 et passim).

7 J. Alber (2016, p. 14) définit le récit non naturel comme présentant « des scénarios et des événements physiquement, logiquement et humainement impossibles [...]». Par "physiquement impossibles», il entend impossible selon les lois fondamentales qui gouvernent le monde physique. Par «logiquement impossibles», impossibles selon les principes de la logique, notamment le principe de non-contradiction. Ces deux définitions sont empruntées à L. Doležel (1998, p. 115) dans sa présentation des mondes naturels et surnaturels de la fiction. Elles apparaissent dès les premiers travaux de narratologie non naturelle de J. Alber (2009, p. 80, cité dans Alber et al., 2010, p. 115 ; Alber \& Heinze, 2011a, p. 4-5). À partir de 2012, J. Alber ajoute « humainement impossibles ", par quoi il entend impossibles compte tenu des limites de la connaissance et des capacités humaines (voir Alber, Nielsen \& Richardson, 2012, p. 351 ; Alber et al., 2012, p. 373 ; Richardson, 2012b, p. 98 ; Alber et al., 2013a, p. 102 ; 2013b, p. 6 ; Alber, 2013b, p. 449 ; 2013d, § 1 et 3).

8 La définition de J. Alber est à la fois plus étroite et plus large que celle de B. Richardson. Ce dernier reconnait lui-même qu'il discute de phénomènes "bizarres ", «inhabituels ", « anormaux », aussi bien que strictement « impossibles ». Pour B. Richardson, le critère permettant de reconnaitre le non-naturel est l'effet de surprise ou de défamiliarisation 
qu'il suscite chez le lecteur ; pour J. Alber, le critère du non-naturel est interne au texte ou plus exactement au monde fictionnel projeté par le texte: c'est la présence de scénarios et d'évènements impossibles, non actualisables dans le monde réel de référence (voir Alber, 2016, p. 3, 16, 29, 32, 41). D'un autre côté, J. Alber a aussi une définition plus large que celle de B. Richardson qui exclut les œuvres non mimétiques conventionnelles, contes de fées, fables animalières, histoires de fantômes, etc., de son champ d'étude. J. Alber, en revanche, les examine en détail (voir p. ex. 2011; 2013a ; 2013b ; et surtout 2016). Il oppose les impossibilités physiques, logiques ou humaines du postmodernisme qui n'ont pas encore été conventionalisées, c'est-à-dire selon lui transformées en schèmes cognitifs de base, et celles qui ont été conventionalisées, comme les animaux parlants dans les fables ou les histoires pour enfants, les fantômes et les vampires dans les romans gothiques, les sorciers dans la littérature de fantasy, le voyage dans le temps dans les romans de science-fiction.

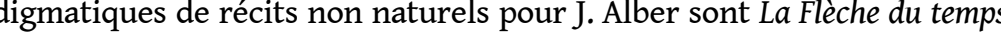
de M. Amis, où le temps s'écoule à l'envers (du futur vers le passé), "The Babysitter ", nouvelle de R. Coover non traduite en français, qui propose des scénarios multiples et contradictoires. On retrouve également Les Enfants de minuit, pour la représentation d'une impossibilité humaine (le narrateur, Saleem Sinai, entend littéralement les pensées des autres personnages).

10 La définition du récit non naturel selon $\mathrm{H}$. S. Nielsen est à première vue proche de celle de J. Alber : «[...] les récits non naturels constituent un sous-ensemble de récits fictionnels pouvant avoir des temporalités, des mondes diégétiques [storyworlds], des représentations de l'esprit [des personnages] ou des actes de narration qui devraient être interprétés comme physiquement, logiquement, mnémoniquement ou psychologiquement impossibles ou implausibles dans les situations de narration du monde réel » (Alber et al., 2013a, p. 104 ; 2013b, p. 6 ; Nielsen, 2013, p. 72; 2014, p. 241) ${ }^{10}$. Les divergences entre H.S. Nielsen et J.Alber apparaitront plus clairement dans la section suivante. H. S. Nielsen ajoute en effet que les récits non naturels " autorisent les lecteurs à les interpréter comme fiables, possibles ou établissant ce qui est vrai [lit. faisant autorité, authoritative], en leur signalant qu'ils doivent modifier leurs stratégies interprétationnelles » (Alber et al., 2013a, p. 104).

On pourrait encore citer la définition des esprits non naturels selon S. Iversen («[...] un esprit non naturel est une conscience [re]présentée qui, dans ses fonctions ou ses réalisations, viole les règles gouvernant le monde possible dont elle fait partie d'une façon qui résiste à la naturalisation ou à la conventionalisation ", 2013b, p. 97), ou selon M. Mäkelä, qui étend la notion jusqu'à englober toutes les représentations littéraires de la conscience ( «[...] nous n'avons pas besoin de recourir à la littérature d'avant-garde pour remarquer que la non-naturalité - ou le type de défi cognitif proprement littéraire - est toujours déjà présente dans les représentations textuelles de la conscience », 2013, p. 133, cité dans Alber et al., 2013b, p. 7).

12 Les exemples paradigmatiques de récits non naturels pour H.S. Nielsen sont des récits comme Glamorama de B. Easton Ellis, qui multiplie les moments où la narration du narrateur devrait être considérée comme impossible ou implausible dans le monde réel de référence. Les exemples paradigmatiques de récits non naturels pour S. Iversen sont «La Métamorphose » de F. Kafka ou Truismes de M. Darrieussecq, qui présentent des esprits métamorphosés, à la fois humains et animaux. Un des problèmes posés par la définition de M. Mäkelä est qu'elle ne permet pas de différencier, dans l'ensemble des 
récits fictionnels, les récits naturels et les récits non naturels. Elle ne permet pas non plus de donner des exemples paradigmatiques de récits non naturels.

\section{Des approches très différentes, voire opposées}

B. Richardson, H. S. Nielsen et S. Iversen se méfient de l'approche cognitive dans l'analyse et l'interprétation des récits non naturels, dans la mesure où ce type d'approche tend à expliquer les récits non naturels en termes de connaissance ordinaire ou d'expérience familière. S. Iversen parle même de "renaturalisation» ("Selon ce type de stratégie interprétative, le travail à la fois du lecteur ordinaire et professionnel est de renaturaliser [...]», 2013b, p. 95). Au contraire, J. Alber plaide pour une approche cognitive du nonnaturel, fondée sur les schèmes (ou cadres, frames) et les scripts qui, gardés en mémoire, sont utilisés dans la vie quotidienne pour établir des liens entre les évènements. Tout son effort consiste à répertorier, puis à exploiter dans ses propres analyses et interprétations, les stratégies utilisables par les lecteurs lorsqu'ils sont confrontés à des évènements ou des scénarios non naturels (stratégies qu'il envisage dans un premier temps comme des stratégies de "naturalisation », voir Alber, 2009, p. 81, 82, 83, avant de parler plutôt de "stratégies de lecture» ou d'«outils de navigation», en réservant le terme «naturalisation » pour la troisième stratégie uniquement, voir p. ex. Alber, 2013b, p. 452, 458, n. $16 ; 2013$ c, p. $49 ; 2014$, p. 274 et n. $18 ; 2016$, p. 51 et p. 237, n. 14). Ces stratégies sont très inspirées d'un article de T. Yacobi portant sur l'interprétation des incongruités dans les récits de fiction (voir 1981). 
Les neuf stratégies de lecture de J. Alber

1. Le mélange des cadres

Cette stratégie consiste à créer de nouveaux cadres en recombinant, élargissant ou modifiant de différentes façons les cadres préexistants.

Exemples : le narrateur mort ; l'arbre qui parle ; Philip Roth, Le Sein.

2. L'explication par les genres littéraires (generification)

Voir le «principe générique » de T. Yacobi (1981, p. 115-116).

Exemples : les animaux parlants dans les fables ou les récits pour enfants; le voyage dans le temps dans les romans de science-fiction.

3. La subjectivisation

Cette stratégie consiste à invoquer les états de conscience particuliers (rêves, fantasmes, hallucinations, etc.) d'un sujet, généralement le narrateur. C'est la seule stratégie de naturalisation pour $\mathrm{J}$. Alber (au sens où elle révèle que ce qui paraissait non naturel est en fait totalement naturel).

Voir le « principe perspectivique » de T. Yacobi (1981, p. 118-119).

4. La mise en avant de la composante thématique

Exemple : les pouvoirs télépathiques du narrateur dans Les Enfants de minuit considérés comme la possibilité d'une compréhension mutuelle entre les différentes ethnies, religions et communautés de l'Inde postcoloniale.

5. La lecture allégorique

Exemple : «La Métamorphose » considérée comme une allégorie de la condition humaine, illustrant le fait que l'homme peut parfois avoir l'impression d'être traité comme de la vermine.

6. La lecture par la satire (satirization) ou la parodie Exemple : Le Sein.

7. Le fait de poser l'existence d'un monde transcendant

Exemples: le paradis, l'enfer, le purgatoire; Comédie de S. Beckett considérée comme l'évocation d'un purgatoire sans purification.

8. Le « faites-le vous-même »

Cette stratégie consiste à utiliser le texte comme un kit de construction pour se fabriquer sa propre histoire.

Voir Ryan (2006, p. 671).

Exemple : «The Babysitter ».

9. La lecture zen

Cette stratégie se fonde sur le rejet des explications précédentes et consiste dans l'acceptation du non-naturel en tant que tel, avec l'inconfort cognitif qu'il peut susciter (p. ex. l'inconfort de la contradiction) ou au contraire avec une réaction de plaisir.

Si la première stratégie de lecture semble juste mais relativement triviale (J. Alber considère lui-même que « ce processus joue un rôle crucial dans tous les cas dans lesquels nous essayons de donner sens au non-naturel ", Alber et al., 2013a, p. 116, n. 8), un certain nombre d'autres stratégies découlent simplement du choix fait par J. Alber d'inclure dans les récits non naturels les récits que $B$. Richardson appelle non mimétiques et qu'il exclut pour sa part de son champ d'étude. On peut rappeler aussi que pour S. Iversen, les récits non naturels (exactement les esprits non naturels dans les récits) sont ceux qui résistent à la naturalisation et à la conventionalisation, termes qui correspondent exactement en 
contexte à la troisième et à la deuxième (associée à la cinquième) stratégie de lecture de $\mathrm{J}$. Alber. J. Alber et $\mathrm{S}$. Iversen se séparent également en ce qui concerne l'interprétation de «La Métamorphose » qui, selon le premier, peut se prêter à une lecture allégorique (voir Alber, 2011, p. 56), alors que le second pose au contraire qu'elle ne le peut pas facilement et que, de toute façon, son intérêt est ailleurs (voir Iversen, 2013b, p. 106; voir aussi Richardson, 2016b, p. 507).

H. S. Nielsen revendique la légitimité de ce qu'il appelle des stratégies de lecture «non naturalisantes » des récits non naturels : «[...] le lecteur a la possibilité de tenter de maximiser la pertinence en appliquant un ensemble de règles interprétationnelles qualitativement différent. Par exemple, il peut stratégiquement considérer qu'il y a du sens à se fier à des données du récit que le narrateur à la première personne [sic] ne peut en aucun cas connaître " (2011b, p. 79) ; «Une approche non naturelle [...] admet que le lecteur puisse se représenter de telles situations comme des descriptions de l'univers fictionnel fiables, vraies [authoritative], factuelles [matter-of-fact]» $(2014$, p. 241) ; «[...] une lecture non naturalisante est un choix interprétationnel qui, à la différence des lectures naturalisantes, ne suppose pas que les conditions et limitations du monde réel doivent s'appliquer à tous les récits de fiction, qu'il s'agisse de logique, de physique, de temps, d'énonciation, de cadrage conceptuel [framing], etc. » (ibid.). À la différence des stratégies de lecture de J. Alber, celles de H. S. Nielsen consistent surtout dans le fait d'écarter des interprétations naturalisantes qui s'avèreraient non pertinentes (p.ex. l'interprétation par la non-fiabilité du narrateur dans certains passages de récits à la première personne). J. Alber reproche d'ailleurs à H.S. Nielsen de rester en deçà de l'interprétation ou du processus de fabrication de sens tel qu'il l'entend lui-même (voir Alber, 2013b, p. 455 ; 2014, p. 261, 265 ; 2016, p. 18).

La dernière stratégie de lecture de J. Alber, la lecture zen, peut être considérée comme une façon d'intégrer dans le système la dimension critique contenue notamment dans la description des stratégies de lecture non naturalisantes de H. S. Nielsen (voir Alber, 2009, p. 83 et 2013b, p. 454, etc.). Il est à noter cependant que cette stratégie n'est jamais évoquée par H. S. Nielsen. Quant à B. Richardson, il dénie que H. S. Nielsen, S. Iversen et lui-même aient une approche « de type zen » (2016a, p. 401).

\section{Quelques réalisations de la narratologie non naturelle}

B. Richardson a proposé une typologie des temps non naturels, souvent citée dans les travaux des narratologues non naturels (voir Richardson, 2002, repris dans 2011a, p. 59-60 ; 2012a, p. 77-78 ; 2013, p. 21 ; 2015, p. 56-57 ; 2017a, p. 196-199 ; cité dans Alber et al., 2010, p. 115, 116 ; Alber \& Heinze, 2011a, p. 7-8 ; Heinze, 2013, p. 32, n. 1, 40 ; Alber, 2016, p. 149). Selon B. Richardson, les temporalités fictionnelles peuvent être : circulaires (comme dans «Le Cercle» de V. Nabokov); contradictoires (comme dans "The Babysitter »); antinomiques (comme dans La Flèche du temps); différentielles (lorsque le temps s'écoule à une vitesse différente pour différents personnages, comme dans Orlando de V. Woolf ou Cloud Nine de C. Churchill); confondues ou mêlées (comme dans Passacaille de R. Pinget) ; duelles ou multiples, ou encore s'écoulant selon des lois inconnues (comme "un minuit long de six cent trente-cinq jours» dans le chapitre des Sundarbans des Enfants de minuit) ${ }^{11}$. Dans tous ces cas, B. Richardson constate que les catégories narratologiques héritées de G. Genette (ordre, durée, fréquence, mais aussi dans certains 
cas, analepse, prolepse, etc.) ne peuvent plus s'appliquer ou ne s'appliquent qu'avec des réserves considérables.

J. Alber a consacré un article aux espaces non naturels, c'est-à-dire, selon sa définition, aux espaces physiquement ou logiquement impossibles (voir 2013c, repris dans 2016, chap.5). Un bon exemple en est apporté par la maison de La Maison des feuilles de M.Z. Danielewski: maison dont l'architecture intérieure se transforme pendant un voyage de ses propriétaires, dont la largeur intérieure se révèle plus grande que la largeur telle qu'elle peut être mesurée à l'extérieur, dont certains couloirs changent de taille, deviennent des labyrinthes, dans laquelle les boussoles sont inutilisables, etc. J. Alber propose une explication de l'espace non naturel de la maison qui utilise la cinquième stratégie de lecture, la lecture allégorique. Selon lui, la maison signifie l'absurdité ou le néant qui envahit potentiellement toutes les relations humaines. Le labyrinthe de la maison met fin à la vie sexuelle de $\mathrm{K}$. et $\mathrm{W}$. Navidson et mène à "l'impatience, la frustration et une aliénation familiale croissante " (Danielewski, cité dans Alber, 2013c, p. 61 et 2016, p. 190). La maison devient la représentation d'un monde hostile qui détruit systématiquement les relations interpersonnelles. On peut noter que le roman présente lui-même l'explication par la subjectivité d'un personnage (troisième stratégie de lecture) pour la rejeter ensuite : «Certains ont suggéré que les horreurs que Navidson a rencontrées dans cette maison étaient simplement des manifestations de sa propre psyché confuse », etc. (Danielewski, 2015, p. 21).

S. Iversen est connu pour ses travaux sur les esprits non naturels (voir 2011, 2013a et 2013b). Dans Iversen (2013b), il concentre son attention sur un cas particulier, qui est "l'esprit métamorphosé ", abréviation pour l'esprit de l'humain métamorphosé en animal, comme dans « La Métamorphose » (mais l'exemple qu'il choisit de développer est celui de Truismes). Il montre que " cette forme de récit bouleverse la distinction entre l'esprit en tant que phénomène intramental et l'esprit en tant que phénomène social d'une façon instructive [...]» (ibid., p. 109). Là où on s'attendrait à trouver des pensées, des sentiments, des motivations intérieures, on rencontre un langage public, des expressions communes, des clichés ; au contraire, là où on s'attendrait à des phénomènes de cognition sociale, par exemple la reconnaissance d'émotions faciales, on rencontre des réactions de peur et de rejet devant l'apparence bizarre présentée par les protagonistes.

H. S. Nielsen s'est essentiellement intéressé aux actes de narration non naturels. Il s'agit d'ailleurs plutôt d'actes ou de modes de narration "bizarres", "inhabituels", " anormaux ", plutôt que strictement " impossibles ", comme le souligne cette réflexion sur la narration à la première personne et au présent :

Il est vrai qu'il n'y a aucune « occasion de narration » et c'est une situation un peu paradoxale, mais décrire cela comme le fait que le narrateur " réalise l'impossible vivre et raconter en même temps » fait courir le risque de placer le paradoxe et l'impossibilité au niveau du monde de l'histoire, comme s'il s'agissait d'une histoire à propos d'un personnage capable de l'impossible. Je préfèrerais dire (et je pense que c'est probablement aussi ce que Phelan veut dire) que nous devons simplement accepter cet acte de narration bien que ce soit un acte narratif impossible dans le monde réel. (Nielsen 2011a, p. 65 ; les citations sont empruntées à Phelan, 1994, p. 233-234.)

Narration à la première personne et au présent (ou narration au "présent fictionnel ", selon l'expression de D. Cohn [2001, p. 164]), narration à la deuxième personne, accès aux pensées d'autrui (je dirais d'ailleurs plutôt: représentation des pensées d'autrui) dans le récit à la première personne : tels sont quelques-uns des sujets abordés dans les articles 
de H. S. Nielsen (voir 2010 ; 2011a ; 2011b ; 2011c ; 2013 ; 2014). À propos de l'accès aux pensées d'autrui dans le récit à la première personne, il convient en effet de distinguer à nouveau les récits dans lesquels l'impossibilité est située au niveau du monde de l'histoire, où il s'agit effectivement d'une histoire à propos d'un personnage capable de l'impossible (p. ex. S. Sinai dans Les Enfants de minuit) et les récits qui nous donnent accès aux pensées de personnages autres que le narrateur, comme si les contraintes liées aux choix de la narration à la première personne étaient suspendues ou comme sous l'effet d'une opération qu'on pourrait décrire comme étant de l'ordre du montage (p. ex. les dernières pensées de Bergotte mourant dans La Prisonnière de M. Proust ou celles des passagers d'un avion en train d'exploser dans Glamorama, ou encore les chapitres XXXVII et XXXVIII de Moby-Dick d'H. Melville). H.S. Nielsen ne s'intéresse qu'à la deuxième catégorie de récits. (Je précise qu'il n'utilise pas le terme de montage ni l'hypothèse, qui sont de moi.) Dans ses analyses, H. S. Nielsen revalorise le rôle de l'auteur, totalement occulté par la prééminence du narrateur et de la communication entre le narrateur et le narrataire dans la narratologie.

Qu'il s'agisse de temps, d'espace, de personnages ou de narrateurs et d'actes de narration non naturels, la particularité du travail de J. Alber (2016) est de relier les éléments non naturels des récits postmodernistes à des actualisations plus anciennes de ces mêmes éléments, conventionalisées par leur association à des genres littéraires spécifiques (fables animalières, épopées médiévales, «romans de circulation » du XviII ${ }^{\mathrm{e}}$ siècle, dont les narrateurs sont des objets, romans gothiques, romans de science-fiction, etc.).

\section{Problèmes de la narratologie non naturelle}

Je ne m'étends pas sur les problèmes qui ont déjà été rencontrés : les contradictions, les zones de non-recouvrement, les divergences entre les définitions et les approches des récits non naturels chez les différents représentants de la narratologie non naturelle. On aura peut-être remarqué aussi des problèmes dans la définition des récits qui servent de repoussoirs : par exemple, chez B. Richardson, les «récits non fictionnels » et les récits (fictionnels) mimétiques ou réalistes (exactement, qui se conforment aux «attentes mimétiques et [aux] pratiques du réalisme »), sans même parler des récits non mimétiques conventionnels; chez H.S. Nielsen, les récits naturels au sens des récits produits naturellement, c'est-à-dire spontanément, dans la conversation ordinaire. Ces types de récits ne se recouvrent pas non plus. On pourrait dire, en reprenant une distinction de P.K. Hansen, que la narratologie non naturelle est concernée par deux phénomènes ou ensembles de phénomènes :

- les récits qui s'écartent du modèle des récits naturels conversationnels, tel qu'il apparait par exemple dans M. Fludernik (1996) ou D. Herman (2009);

- les récits « du non-naturel », qui présentent des scénarios et des évènements, y compris des scénarios de narration, impossibles dans le monde réel de référence (voir Hansen, 2011, p. 163).

Ce dernier point pose le problème de l'amalgame qui peut être fait entre les deux ensembles de phénomènes. J'en donnerai un exemple à propos du narrateur omniscient. Un autre problème est celui de la prééminence du modèle des récits naturels conversationnels, dont le champ de pertinence mériterait d'être redéfini, mais je ne l'aborderai pas ici. 
Le thème de l'omniscience ou du narrateur omniscient apparait dès les premiers travaux de la narratologie non naturelle, au titre des «formes et techniques» qui ont été "conventionalisées" au cours du temps, sans avoir été pour autant "naturalisées" (Alber et al., 2010, p. 131). La distinction entre conventionalisation et naturalisation est retravaillée dans H. S. Nielsen (2011b, p. 84-86) et présentée à nouveau dans J. Alber, H. S. Nielsen et B. Richardson (2012, p. 352) :

[...] même si une technique narrative est hautement divergente par rapport aux paramètres de la vie réelle, elle peut être (ou être devenue) hautement conventionnelle. Un bon exemple en est apporté par les récits racontés par ce qui est souvent désigné comme un narrateur à la troisième personne [sic] "omniscient », qui est omniprésent, omnitemporel et capable de donner accès aux esprits des personnages. [...] Les récits appartenant à cette catégorie sont non naturels dans la mesure précise où ils ne correspondent à aucune situation de narration de la vie réelle, mais ils ne sont plus considérés comme expérimentaux ou non conventionnels.

Dans cette citation, la périphrase "ce qui est souvent désigné comme», ainsi que les guillemets entourant le terme "omniscient » trahissent une certaine gêne vis-à-vis de cette appellation. Elle disparait pourtant complètement dans d'autres occurrences (voir p. ex. Alber et al., 2013b, p. 6 ; Alber, 2013d, § 2 et 13; Richardson, 2015, p. 35-36, 41; Alber, 2016, p. 88-103).

La narration omnisciente n'a pendant longtemps posé aucun problème particulier à la théorie narrative. Traditionnellement, cette théorie ne distinguait le narrateur de l'auteur ou ne concevait un narrateur fictionnel que dans le cas du roman-mémoires ou roman à la première personne au sens originel du terme (voir Patron, 2016, p. 15-19). Dans cette théorie, le narrateur n'est évidemment pas omniscient: la narration à la première personne représente l'antithèse de la narration omnisciente. La narration du roman à la troisième personne est attribuée à l'auteur lui-même. C'est donc à lui qu'est appliqué le qualificatif «omniscient ». Cependant, cette omniscience doit être considérée comme un effet du texte lui-même et de la nature des informations qu'il contient; comme l'écrit G. Genette (2007, p. 348), «l'auteur n'a rien à "savoir", puisqu'il invente tout ». Le terme «omniscient» n'est devenu problématique que lorsque les narratologues, G. Genette avec certaines précautions mais d'autres sans, se sont mis à l'appliquer à un narrateur fictionnel censé raconter des faits réels. Le narrateur omniscient doit en effet être considéré comme la conséquence du postulat de la présence d'un narrateur fictionnel dans tous les récits de fiction. Mais quel type d'entité représente-t-il, d'où vient son omniscience ? Comment peut-il être à la fois fictionnel, c'est-à-dire appartenant au même monde fictionnel que les personnages, et omniscient?

Le problème de H. S. Nielsen, J. Alber et des autres narratologues non naturels est qu'ils confondent des éléments non naturels inventés par les auteurs de récits de fiction et le narrateur omniscient, qui est une invention de la narratologie («[...] des scénarios et des événements non naturels qui ont déjà été conventionalisés et transformés en catégorie cognitive [...] tels "l'animal parlant", le narrateur "omniscient" [...]", Alber et al., 2010, p. 131 ; voir aussi Alber et al., 2013b, p. 6 ; Alber, 2013a, p. 2138 et n. 5). Si cela a du sens de parler d'éléments qui ont été conventionalisés sans pour autant avoir été naturalisés pour l'animal parlant des fables animalières, cela en a beaucoup moins pour le (prétendu) narrateur omniscient. «narrateur omniscient » : elle est considérée chez lui comme une entité existant dans le 
monde fictionnel et dotée de propriétés, comme n'importe quelle autre entité fictionnelle :

En dépit de la volonté de s'affranchir du surnaturalisme de la romance, le narrateur omniscient des romans réalistes et le médium narratif des récits en mode réflecteur du modernisme littéraire impliquent aussi un certain degré de magie. Comme les magiciens (tels Merlin), ces narrateurs ou média narratifs sont capables de télépathie : contrairement aux agents du monde réel, ils peuvent littéralement lire les esprits des autres personnages (voir aussi Alber, 2013a). (Alber, 2013d, § 13.)

Comme les magiciens et les sorcières, les narrateurs ou les voix à la troisième personne dans ces types de fiction [modernistes] sont capables d'omnimentalité : ils ont accès aux esprits des autres personnages, satisfaisant ainsi le désir commun à tous les humains de connaître les pensées et les sentiments de leurs semblables. À cet égard, ces types de fiction anciens transcendent les paramètres du monde réel exactement comme les récits postmodernistes le font. (Alber, 2016, p. 103.)

Dans cette hypothèse, des romans réalistes comme ceux de G. Flaubert ou un roman moderniste comme Mrs Dalloway de V. Woolf, souvent cité par J. Alber (voir 2011, p. 57-58 ; 2013a, p. 146-147 ; 2016, p. 97-98), deviennent des proches cousins des feéries médiévales, des poèmes héroïques de la Renaissance italienne - voire, si l'on extrapole un peu, des Bijoux indiscrets de D. Diderot ${ }^{12}$.

Chez J. Alber, l'exemple des Enfants de Minuit, dans lequel il y a un narrateur fictionnel, doté de la propriété fictionnelle d'entendre littéralement les pensées des autres personnages ( $[. .$.$] je répète, sans aucune honte, mon incroyable affirmation : après un$ curieux accident dans un coffre à linge, je suis devenu une sorte de poste de radio ", Rushdie, 1989, p. 241), cet exemple est systématiquement confondu avec des exemples de romans réalistes ou modernistes, prétendument dotés ou dotés par la narratologie d'un narrateur omniscient, qui n'a pas de réalité imaginative pour le lecteur (voir Alber, 2013a, p. $137,138,143,147 ; 2016$, p. 25, 81, 88).

La narratologie non naturelle doit s'employer à clarifier son rapport à la notion de fiction et à celle de vérité fictionnelle (il est vrai fictionnellement que Les Enfants de minuit est raconté par un narrateur télépathe, "une sorte de poste de radio "; il n'est pas vrai fictionnellement que Mrs Dalloway est raconté par un narrateur omniscient). Elle doit être plus vigilante et critique, et tenir compte de la complexité de ce que G. Currie (2010, p. 59) a appelé la « limitation de la correspondance représentationnelle », c'est-à-dire le fait que « [p]our une œuvre représentationnelle donnée, seuls certains traits de la représentation servent à représenter des traits des choses représentées » (on l'a vu dans le cas de la narration à la première personne et au présent mais on pourrait citer d'autres exemples). Je pense aussi qu'il y aurait tout un travail à faire sur la réception des récits non naturels en termes de remplissage des blancs du texte mais aussi d'ignorance de certaines lacunes, ces récits entretenant selon moi un rapport particulier avec la règle de l'incomplétude des mondes de fiction.

33 La narratologie non naturelle doit se montrer plus vigilante aussi dans sa volonté de diachroniser. E. Von Contzen a raison de dire qu'à moins qu'il n'y ait des liens intertextuels directs ou indirects, les récits prémodernes n'« anticipent » pas (c'est le mot de J. Alber) les œuvres plus tardives. L'idée d'anticipation implique à la fois la téléologie et les jugements de valeur (voir Von Contzen, 2017, notamment p. 9). On ne peut pas non plus se contenter d'identifier des éléments non naturels, comme les magiciens et les fées mais aussi les miracles dans les récits médiévaux, indépendamment de leur inscription dans un contexte historique et culturel donné (voir ibid.). 

choix du non-naturel et l'idéologie au sens large du terme, aux xxe et xxI siècles (voir Richardson, 2015, chap. 7 ; Richardson, 2017b). Elle a encore peu de réalisations dans le domaine de l'étude du non-naturel à travers les médias : films, bandes dessinées, romans graphiques, jeux vidéo, etc. ${ }^{13}$. Telles sont quelques-unes des perspectives qui se dessinent pour l'avenir.

\section{BIBLIOGRAPHIE}

ALBER, J. (2009). « Impossible Storyworlds - and What To Do With Them ». Storyworlds 1 (1), p. 79-96.

ALBER, J. (2011). « The Diachronic Development of Unnaturalness: A New View on Genre ». In : Alber, J. \& Heinze, R. (eds), Unnatural Narratives-Unnatural Narratology. Berlin : De Gruyter, p. 41-67.

ALBER, J. (2013a). « Pre-Postmodernist Manifestations of the Un Natural: Instances of Expanded Consciousness in "Omniscient" Narration and Reflector-Mode Narratives ». Zeitschrift für Anglistik und Amerikanistik 61 (2), p. 137-153.

ALBER, J. (2013b). « Unnatural Narratology: The Systematic Study of Anti-Mimeticism ». Literature Compass 10 (5), p. 449-460.

ALBER, J. (2013c). « Unnatural Spaces and Narrative Worlds ». In : Alber, J. et al. (eds), A Poetics of Unnatural Narrative. Columbus : The Ohio State University Press, p. 45-66.

ALBER, J. (2013d). « Unnatural Narrative ». In : P. Hühn et al. (eds), The Living Handbook of Narratology. En ligne : http://www.lhn.unihamburg.de/article/unnatural-narrative.

ALBER, J. (2014). « Postmodernist Impossibilities, the Creation of New Cognitive Frames, and Attempts at interpretation ». In : Alber, J. \& Hansen, P. K. (eds), Beyond Classical Narration : Transmedial and Unnatural Challenges. Berlin : De Gruyter, p. 261-280.

ALBER, J. (2016). Unnatural Narrative: Impossible Worlds in Fiction and Drama. Lincoln : University of Nebraska Press.

ALBER, J. \& HEINZE, R. (2011a). «Introduction ». In : Alber, J. \& Heinze, R. (eds), Unnatural Narratives-Unnatural Narratology. Berlin : De Gruyter, p. 1-19.

ALBER, J. \& HEINZE, R. (eds) (2011b). Unnatural Narratives-Unnatural Narratology. Berlin : De Gruyter. ALBER, J. \& RICHARDSON, B. (eds) (à paraître). Unnatural Narratives: Critical Theory and Cultural Studies. Columbus : The Ohio State University Press.

ALBer, J., CARACCIOlO, M., IVERSEN, S., KUKKONEN, K., \& NielSEN, H. S. (eds) (à paraître). « Unnatural and Cognitive Perspectives on Literary Studies: A Theory Crossover ». Poetics Today, 39 (1-2).

ALBER, J., IVERSEN, S., NIELSEN, H. S. \& RICHARDSON, B. (2010). « Unnatural Narratives, Unnatural Narratology: Beyond Mimetic Models ». Narrative 18.2, p. 113-136.

Pratiques, $181-182$ | 2019 
ALBER, J., IVERSEN, S., NIELSEN, H. S. \& RICHARDSON, B. (2012). « What Is Unnatural about Unnatural Narratology? A Response to Monika Fludernik ». Narrative 20 (3), p. 371-382.

ALBER, J., IVERSEN, S., NIELSEN, H. S. \& RICHARDSON, B. (2013a). « What Really Is Unnatural Narratology?». Storyworlds 5, p. 101-118.

ALBER, J., IVERSEN, S., NIELSEN, H. S. \& RICHARDSON, B. (2013b). « Introduction ». In Alber, J. et al. (eds), A Poetics of Unnatural Narrative. Columbus : The Ohio State University Press, p. 1-15.

ALBER, J., NIELSEN, H. S. \& RICHARDSON, B. (2012). « Unnatural Voices, Minds, and Narration ». In : J. Bray et al. (eds), The Routledge Companion of Experimental Literature. London : Routledge, p. 351-367. ALBER, J., NIELSEN, H. S. \& RICHARDSON, B. (eds) (2013). A Poetics of Unnatural Narrative. Columbus : The Ohio State University Press.

COHN, D. (2001) [1999]. Le Propre de la fiction. Trad. par C. Harry-Schaeffer. Paris : Éditions du Seuil. CURRIE, G. (2010). Narrative and Narrators: A Philosophy of Stories. Oxford : Oxford University Press. DANIELEWSKI, M. Z. (2015) [2000]. La Maison des feuilles. Trad. par Christophe Claro. Paris : Éditions du Seuil.

DOLEŽEL, L. (1998). Heterocosmica: Fiction and Possible Worlds. Baltimore : Johns Hopkins University Press.

FLUDERNIK, M. (1996). Towards a « Natural » Narratology. London : Routledge.

FLUDERNIK, M. (2012). « How Natural Is "Unnatural Narratology"; or What is Unnatural about Unnatural Narratology?». Narrative 20 (3), p. 357-370.

GENETTE, G. (2007) [xxxx]. Discours du récit. Paris : Éditions du Seuil.

HANSEN, P. K. (2011). « Backmasked Messages: On the Fabula Construction in Episodically Reversed Narratives ». In : Alber, J. \& Heinze, R. (eds), Unnatural Narratives-Unnatural Narratology. Berlin : De Gruyter, p. 162-185.

HEINZE, R. (2013). « The Whirligig of Time: Towards a Poetics of Unnatural Temporality ». In Alber, J. et al. (eds), A Poetics of Unnatural Narrative. Columbus, The Ohio State University Press, p. 31-44. HERMAN, D. (2009). Basic Elements of Narrative. Malden : Wiley-Blackwell.

HERMAN, D. et al. (2012). Narrative Theory: Core Concepts and Critical Debates. Columbus : The Ohio State University Press.

IVERSEN, S. (2011). « "In Flaming Flames”: Crises of Experientiality in Non-Fictional Narratives ». In : Alber, J. \& Heinze, R. (eds), Unnatural Narratives-Unnatural Narratology. Berlin : De Gruyter, p. 89-103.

IVERSEN, S. (2013a). « Broken or Unnatural? On the Distinction of Fiction in Non-Conventional First Person Narration ». In : Hyvärinen, M. et al. (eds), The Travelling Concept of Narrative. Amsterdam : John Benjamins Publishing, p. 141-162.

IVERSEN, S. (2013b). « Unnatural Minds ». In : Alber, J. et al. (eds), A Poetics of Unnatural Narrative. Columbus : The Ohio State University Press, p. 94-112.

KLAUK, T. \& KÖPPE, T. (2013). « Reassessing Unnatural Narratology : Problems and Prospects ». Storyworlds 5, p. 77-100. 
MÄKELÄ, M. (2013). « Cycles of Narrative Necessity: Suspect Tellers and the Textuality of Fictional Minds ». In L. Bernaerts et al. (eds), Stories and Minds: Cognitive Approaches to Literary Narratives. Lincoln : University of Nebraska Press, p. 129-151.

NIELSEN, H. S. (2010). « Natural Authors, Unnatural Narration ». In J. Alber \& M. Fludernik (eds), Postclassical Narratology: Approaches and Analyses. Columbus : The Ohio State University Press, p. 275-301.

NIELSEN, H. S. (2011a). « Fictional Voices? Strange Voices? Unnatural Voices ? ». In : Hansen, P. K. et al. (eds), Strange Voices in Narrative Fiction. Berlin : De Gruyter, p. 55-82.

NIELSEN, H. S. (2011b). « Unnatural Narratology, Impersonal Voices, Real Authors, and NonCommunicative Narration ». In : Alber, J. \& Heinze, R. (eds), Unnatural Narratives-Unnatural Narratology. Berlin : De Gruyter, p. 71-88.

NIELSEN, H. S. (2011c). «Theory and Interpretation, Narration and Communication, Authors and Narrators - James Frey's A Million Little Pieces as a Test Case ». In : Patron, S. (éd.), Théorie, analyse, interprétation des récits/Theory, Analysis, Interpretation of Narratives. Bern : Peter Lang, p. 73-92.

NIELSEN, H. S. (2013). « Naturalizing and Unnaturalizing Strategies : Focalization Revisited ». In : Alber, J. et al. (eds), A Poetics of Unnatural Narrative. Columbus : The Ohio State University Press, p. 67-93.

NIELSEn, H. S. (2014). « The Unnatural in E. A. Poe's “The Oval Portrait” ». In : Alber, J. \& Hansen, P. K. (eds), Beyond Classical Narration: Transmedial and Unnatural Challenges. Berlin : De Gruyter, p. 239-260.

OLSEN, J.-A. (2004). L'Esprit du roman. Cuvre, fiction et récit. Bern : Peter Lang.

PATRON, S. (2012). « Phrases sans parole. À propos d'une histoire d'amour du $\mathrm{xx}^{\mathrm{e}}$ siècle ». CMLF 2012, $3^{\mathrm{e}}$ Congrès mondial de linguistique française, Lyon, 4-7 juil. En ligne : http://www.shsconferences.org/index.php?option=com_article\&access=doi\&doi=10.1051/ shsconf/20120100206\&Itemid $=129$.

PATRON, S. (2016). Le Narrateur. Un problème de théorie narrative. Limoges : Lambert-Lucas.

PATRON, S. (éd.) (2018). Introduction à la narratologie postclassique. Les nouvelles directions de la recherche sur le récit. Villeneuve d'Ascq : Presses universitaires du Septentrion.

PHELAN, J. (1994). « Present Tense Narration, Mimesis, the Narrative Norm, and the Positioning of the Reader in Waiting for the Barbarians ». In : Phelan, J. \& Rabinowitz, P. J. (eds), Understanding Narrative. Columbus : The Ohio State University Press, p. 222-245.

RICHARDSON, B. (2002). «Beyond Story and Discourse : Narrative Time in Postmodern and Nonmimetic Fiction ». In : Richardson, B. (ed.), Narrative Dynamics: Essays on Time, Plot, Closure, and Frames. Columbus : The Ohio State, p. 47-63.

RICHARDSON, B. (2006). Unnatural Voices: Extreme Narration in Modern and Contemporary Fiction. Columbus : The Ohio State University Press.

RICHARDSON, B. (2011). « Narrative Theory, Methodology, and the Unusual Text ». In : Patron, S. (éd.), Théorie, analyse, interprétation des récits/Theory, Analysis, Interpretation of Narratives. Bern : Peter Lang, p. 57-71.

RICHARDSON, B. (2012a). « s.t. ». In : Herman, D. et al., Narrative Theory: Core Concepts and Critical Debates. Columbus : The Ohio State University Press. 
RICHARDSON, B. (2012b). « Unnatural Narratology: Basic Concepts and Recent Work ». Diegesis 1 (1), p. 95-103. En ligne : https://www.diegesis.uni-wuppertal.de/index.php/diegesis/article/ view/112/119.

RICHARDSON, B. (2013). «Unnatural Stories and Sequences ». In : Alber, J. et al. (eds), A Poetics of Unnatural Narrative. Columbus : The Ohio State University Press, p. 16-30.

RICHARDSON, B. (2015). Unnatural Narrative: Theory, History, and Practice. Columbus : The Ohio State University Press.

RICHARDSON, B. (2016a). « Unnatural Narrative Theory ». Style 50 (4), p. 385-405.

RICHARDSON, B. (2016b). « Rejoinders to the Respondents ». Style 50 (4), p. 492-513.

RICHARDSON, B. (2017a). « Unnatural Narrative Theory: A Paradoxical Paradigm ». In : Hansen, P. K. et al. (eds), Emerging Vectors of Narratology. Berlin : De Gruyter, p. 193-206.

RICHARDSON, B. (ed.) (2017b). « Special Section on Feminist Fiction and Unnatural Narrative Theory ». Storyworlds 8 (2).

RICHARDSON, B. (2018). « De la narratologie non naturelle ». Trad. par S. Patron, à partir d'une première version de Catherine Favier Kelly. In : Patron, S. (éd.), Introduction à la narratologie postclassique. Les nouvelles directions de la recherche sur le récit. Villeneuve d'Ascq : Presses universitaires du Septentrion, p. 167-181.

RICHARDSON, B. (ed.) (à paraître). « Unnatural Narratives: Theories and Practices ». Frontiers of Narrative Studies 4 (1).

RUSHDIE, S. (1989) [1981]. Les Enfants de minuit. Trad. de l'anglais par J. Guiloineau. Paris : Librairie générale française.

RYAN, M.-L. (2006). «From Parallel Universes to Possible Words: Ontological Pluralism in Physics, Narratology, and Narrative ». Poetics Today 27 (4), p. 633-674.

STYLE (2016). 50 (4).

VON CONTZEN, E. (2017). « Unnatural Narratology and Premodern Narratives: Historicizing a Form ». Journal of Literary Semantics 46 (1), p. 1- 23.

YACOBI, T. (1981). « Fictional Reliability as a Communicative Problem ». Poetics Today 2 (2), p. $113-126$

\section{NOTES}

1. Pour une introduction à la narratologie postclassique en français, voir Patron, éd. (2018). L'ouvrage contient un article de Brian Richardson sur la narratologie non naturelle. Les références complètes des ouvrages et articles sont données dans la bibliographie.

2. Toutes les traductions de l'anglais sont de moi.

3. L'adjectif «non naturel » avait déjà été utilisé dans ce sens par Richardson (voir 2006, notamment p. 37 et index p. 165 : « récit, naturel », « récit, non naturel »).

4. La page correspondante est actuellement hébergée par le site de l'Université d'Aarhus, Danemark, et on peut également $y$ accéder via : http://projects.au.dk/ narrativeresearchlab/unnatural/. 
5. Voir www.unnaturalnarratology.com, rubrique « Presentations » (période 2008-2012).

6. Cet article a suscité des réponses (voir Fludernik, 2012 ; Klauk \& Köppe, 2013), qui à leur tour ont permis aux narratologues non naturels de clarifier leurs positions (voir Alber et al., 2012 et 2013a).

7. Un troisième est à paraître (voir Alber \& Richardson, à paraître).

8. Un numéro double de la revue Poetics Today et un numéro de la revue Frontiers of Narrative Studies sont à paraître (voir Alber et al., à paraître ; Richardson, à paraître).

9. Il s'agit ici de la dernière formulation de la définition de B. Richardson, qui en a connu d'autres, plus fréquemment citées.

10. Cette définition apparaissait déjà dans Nielsen (2010, p. 276, n. 3), mais limitée aux actes de narration.

11. Je me permets d'ajouter la temporalité trouée, lacunaire, de «Cinq ans de vie » de M. Benedetti, où il manque cinq ans dans la vie du protagoniste (voir Patron, 2012).

12. J'emprunte ce rapprochement à J.-A. Olsen (2004, p. 301). Je rappelle que l'invisibilité et l'omnimentalité de Mangogul dans Les Bijoux indiscrets s'expliquent par les pouvoirs magiques de l'anneau dont il est devenu le propriétaire.

13. Certains articles sont à paraître sur ces sujets : voir Alber \& Richardson (à paraître).

\section{RÉSUMÉS}

L'étude des récits non naturels (unnatural narratives), ou narratologie non naturelle (unnatural narratology), est l'un des derniers courants apparus au sein de la narratologie postclassique. Cette approche parait novatrice et prometteuse car elle rejoint plusieurs préoccupations actuelles associées à la théorie, à l'analyse et à l'interprétation des récits de fiction. Elle est aussi intensément discutée dans les milieux narratologiques. Loin d'être une approche unifiée, la narratologie non naturelle se compose de différentes tendances, basées sur des définitions légèrement différentes des récits non naturels, prônant des approches parfois très différentes, voire opposées, de ces récits. La narratologie non naturelle peut déjà se prévaloir d'apports importants dans l'étude des récits non naturels ou des éléments non naturels dans les récits, généralement négligés dans la ou les narratologies établies. Une étude un peu approfondie fait cependant apparaitre certains problèmes. Un exemple significatif est donné à la fin de l'article.

The study of unnatural narratives, or unnatural narratology, is one of the latest trends that have emerged as part of postclassical narratoloy. This approach seems innovative and promising as it addresses several current concerns associated to theory, analysis and interpretation of fictional narratives. It is also intensely discussed in the narratological milieu. Far from being a unified approach, unnatural narratology consists of different tendencies, based on slightly different definitions of unnatural narratives, advocating sometimes very different and even opposed approaches of these narratives. Unnatural narratology has already made important contributions in the study of unnatural narratives or of unnatural elements in narratives, usually neglected in established narratology or narratologies. Further investigation reveals however certain problems. A significant example is presented at the end of the article. 
INDEX

Mots-clés : récits non naturels, narratologie non naturelle, récits antimimétiques, conventionalisation, approche cognitive, narrateur omniscient

Keywords : unnatural narratives, unnatural narratology, antimimetic narratives, conventionalization, cognitive approach, omniscient narrator

\section{AUTEUR}

\section{SYLVIE PATRON}

Université Paris Diderot, Cérilac, F-75013 Paris, France 